\title{
MCT/LCT/Fish Oil Omega-3 Fatty Acid Lipid Emulsion
}

National Cancer Institute

\section{Source}

National Cancer Institute. MCT/LCT/Fish Oil Omega-3 Fatty Acid Lipid Emulsion. NCI

Thesaurus. Code C79839.

A nutritional lipid emulsion consisting of coconut oil-derived medium chain triglycerides (MCTs), soybean oil-derived long chain triglycerides (LCT), and the fish oil-derived polyunsaturated omega-3 fatty acids. This lipid emulsion supplies essential fatty acids and calories for energy. Omega-3 fatty acids may decrease the production of certain pro-inflammatory cytokines, including interleukin 1 (IL-1), II-6 and tumor necrosis factor (TNF). The MCT s mainly provide calories for energy. In addition to LCTs, MCT s, and omega-3 fatty acids, this lipid emulsion contains egg yolk lecithin, glycerol, and the antioxidant alpha-tocopherol (vitamin E). 